\title{
CACTACEAE DO VALE DO RIO JEQUITINHONHA (MINAS GERAIS)'
}

\author{
Nigel P. Taylor ${ }^{2}$ \\ Daniela C. Zappi ${ }^{3}$
}

Recebido em 10-12-90. Aceito em 10-09-91

RESUMO - Durante as primeiras coletas relacionadas ao Projeto "Cactaceae no Brasil Oriental”, ficou provada a grande diversidade de espércies dessa família na região do vale médio do Rio Jequitinhonha, tendo sido visitadas as localidades de Itinga, Itaobim e Pedra Azul. Foram coletadas 21 espécies distribuídas entre os gêneros Pereskia, Opuntia, Tacinga, Pseudoacanthocereus, Arrojadoa, Brasilicereus, Cereus, Coleocephalocereus, Melocactus, Pilosocereus e Selenicereus, representando as três subfamílias de Cactaceae, cujas afinidades taxonômicas e padrões de distribuição geográfica são discutidos a seguir.

Palavras-chave: Cactaceae, florística, distribuição geográfica, Minas Gerais

ABSTRACT - During an initial visit to collect material for the projected "Cacti of Eastern Brazil", a remarkable great diversity of species of Cactaceae in the region of middle drainage of the Rio Jequitinhonha (mainly in the localities of Itinga, Itaobim and Pedra Azul) was noted. Twenty one species in the genera Pereskia, Opuntia, Tacinga, Pseudocanthocereus, Arrojadoa, Brasilicereus, Cereus, Coloecephalocereus, Melocactus, Pilosocereus e Selenicereus representing all three subfamilies of Cactaceae were recorted. The phytogeographic affinities of the Cactaceae from this region are discussed.

Key-words: Cactaceae, floristics, geographical distribution, Minas Gerais

\section{Introdução}

O centro de diversidade das cactáceas do Brasil Oriental é representado pelas regiões semi-áridas dos estados da Bahia e Minas Gerais, onde estão localizadas importantes bacias fluviais notáveis pelas suas floras ricas em cactáceas endêmicas.

1. Trabalho parcialmente financiado pela National Geographic Society

2. Royal Botanic Gardens, Kew, Richmond, TW9-3AB, Inglaterra

3. Depto. de Botânica da UNESP - Campus de Rio Claro, Av. 24 A, s.n. CEP 13500, Rio Claro, SP. 
Por exemplo, o sistema de drenagem do Rio São Francisco inclui gêneros e subgêneros endêmicos como Espostoopsis (1 sp.) e Facheiroa subg. Facheiroa (2 spp.), além de espécies endêmicas de Melocactus (4), Pilosocereus (4), Discocactus (2), Micranthocereus subg. Siccobaccatus (1), Quiabentia zehntnerii, Brit. \& Ritter, Pereskia stenantha, Ritter e outras. O Rio Jequitinhonha, a NE de Minas Gerais, trata-se de um sistema consideravelmente menor, embora seja também de grande importância em termos de sua flora de cactáceas.

As cabeceiras do Rio Jequitinhonha, que drenam uma ampla região do NE de Minas Gerais, na face Leste da Cadeia do Espinhaço, incluem os habitats do gênero Uebelmannia (c. 4 spp.), cujas afinidades taxonômicas ainda não se encontram esclarecidas, do notável Cipocereus crassisepalus (Bruin. \& Brederoo) Zappi \& Taylor (Hunt \& Taylor eds., 1991), além de Discocactus horstii, D. pseudoinsignis Taylor \& Zappi (D. insignis (sensu auctt.), Brasilicereus markgrafii Backeb. \& Voll. Micranthocereus auriazureus Buin. \& Brederoo, $M$. violaciflorus Buin. e Pilosocereus fulvilanatus (Buin. \& Brederoo) Ritter.

A região média de drenagem deste sistema fluvial, isto é, a área que inclui os municípios de Itinga, Itaobim, Medina, Pedra Azul e Jequitinhonha, não é de modo algum menos interessante.

\section{Material e Métodos}

Dotada de geomorfologia peculiar oriunda de denudamento de rochas plutônicas, inicialmente profundas (King, 1956), a região média do vale do Rio Jequitinhonha possui fisionomia típica de caatinga e apresenta precipitação anual em torno de 900 mm (Rizzini, 1979).

Durante a execução deste trabalho, desenvolvido durante a fase preliminar do Projeto Cactaceae do Brasil Oriental, em outubro-novembro de 1988, foram visitadas várias localidades, entre as quais se destacam:

- Pedra Azul - Medina: predominantemente afloramentos gnáissicos (pontões), associados a caatinga arbustiva e arbórea.

- Itaobim - Itinga: afloramentos gnáissicos e calcáricos (lagedos) resultantes da denudação fluvial, associados à caatinga arbustiva à margem do rio.

- Jequitinhonha (Serra da Areia): região serrana (c. 1000 m s.n.m.) com areal de origem provavelmente quartzítica e vegetação de campo rupestre.

Diversos espécimes das 21 espécies encontradas foram coletados e herborizados seguindo a metodologia tradicional e as porções reprodutivas foram fixadas em álcool 70\%, encontrando-se depositados nos herbários da Univ. São Paulo (SPF) e do Royal Botanic Gardens, Kew (K), e nas respectivas coleções de material fixado. 
A identificação foi baseada principalmente em Ritter (1979) e a delimitação genérica seguiu os conceitos de Taylor \& Zappi (1989). Após a identificação dos espécimes coletados, foram confeccionados mapas de distribuição geográfica para as espécies endêmicas e de distribuição disjunta, utilizando o material coletado e estudo de material depositado nos seguintes herbários: K, SPF, RB, CEPEC.

\section{Resultados e Conclusões}

Foram identificadas 21 espécies de cactáceas na região, cuja lista encontra-se a seguir:
A. subfamília PERESKIOIDEAE
1.Pereskia aureiflora Ritter
2.Pereskia grandifolia Haw. var. violaceae Leuenberger
B. subfamília OPUNTIOIDEAE

3.Opuntia brasiliensis (Willd.) Haw.

4. Opuntia inamoena Schumann

5. Opuntia sp. nov.

6. Tacinga braunii E. Esteves-Pereira

C. subfamília CACTOIDEAE

7. Pseudoacanthocereus brasiliensis (Britton \& Rose) Ritter

8. Arrojadoa penicillata (Guerke) Britton \& Rose

9. Brasilicereus phaecanthus (Guerke) Werdermann

10. Cereus jamacaru DC.

11. Coleocephalocereus aureus Ritter

12. Coleocephalocereus purpureus (Buin. \& Brederoo) Ritter

13. Melocactus bahiensis ssp. am thystinus (Buin. \& Brederoo) Taylor

14. Melocactus ernestii Vaupel

15. Melocactus violaceus Pfeiffer

16. Pilosocereus cenepequei Rizzini \& Mattos-F.

17. Pilosocereus floccosus Byles \& Rowley

18. Pilosocereus magnificus (Buin. \& Brederoo) Ritter

19. Pilosocereus multicostatus Ritter

20. Pilosocereus subsimilis Rizzini \& Mattos-F.

21. Selenicereus setaceus (Salm-Dyck ex DC.) Werdermann

O exame detalhado da flora de cactáceas revelou surpreendente concentração de espécies, com 10 ou mais taxas diferentes crescendo juntos num habitat reduzido, como, por exemplo, num pequeno lageado gnáissico e suas proximidades, a oeste de Itaobim: Pereskia aureiflora, Tacinga braunii, Opuntia inamoiena, Pseudoacanthocereus brasiliensis, Cereus jamacaru, Arrojadoa penicillata, Coleocephalocereus aureus, Melocactus ernestii 
e Melocactus bahiensis ssp. amethystinus.

Poucos quilômetros a oeste de Itaobim, nas proximidades de Itinga, a lista apresentada anteriormente modifica-se: Coleocephalocereus aureus é substituído por $C$. purpureus, surgem Pilosocereus magnificus e P. subsimilis, e Brasilicereus phaeacanthus e Opuntia (Brasiliopuntia) brasiliensis são encontrados ao invés de Tacinga braunii e Pseudoacanthocereus brasiliensis.

Seguindo para Norte de Itaobim, em pontões rochosos aparentemente menos áridos na região de Pedra Azul, é encontrado um maior número de espécies de Pilosocereus, como P. multicostatus, $P$. floccosus e $P$. cenepequei. Ali também ocorrem Pereskia grandifolia vá. violacea, Selenicereus setaceus, Brasilicereus phaeacanthus e Opuntia sp. nov.

São apresentados dois mapas de distribuição geográfica para ilustrar as espécies com padrões de distribuição mais interessantes. A Figura 1 representa a região de coleta e encontram-se plotadas as espécies endêmicas para a região. Na Figura 2, pode-se observar as espécies com distribuição disjunta na região leste do Brasil, estando destacada a Cadeia do Espinhaço.

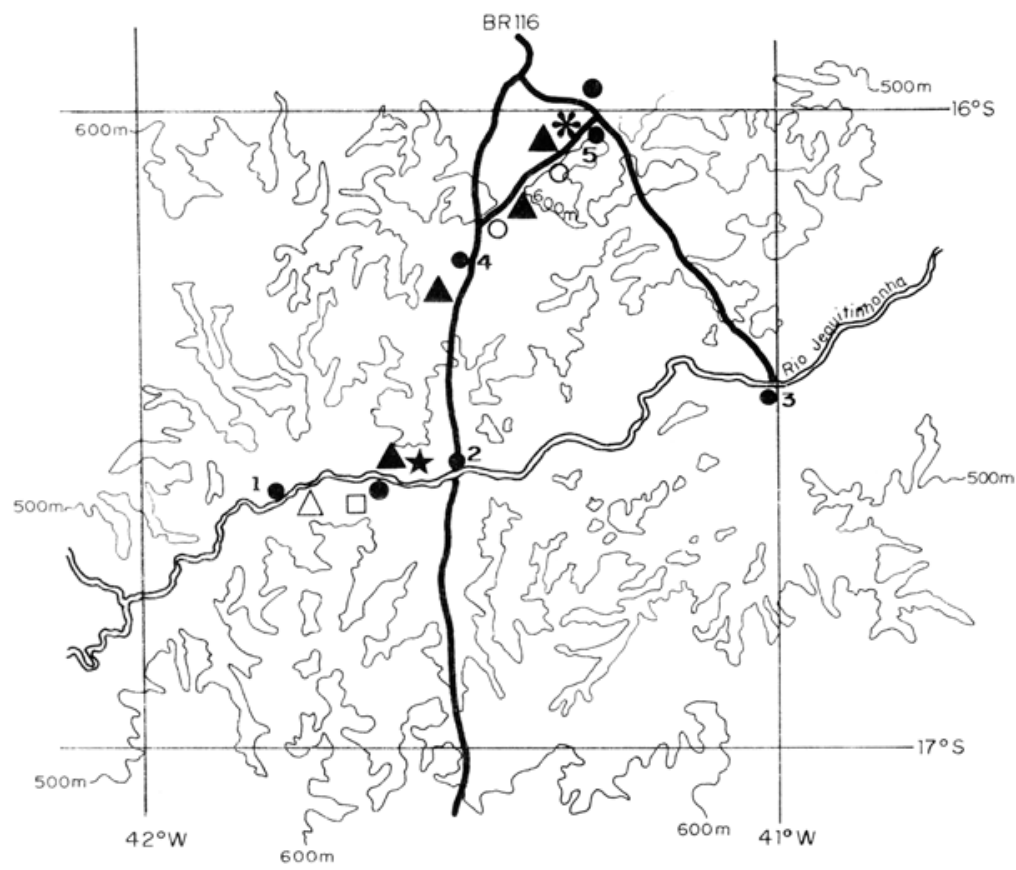

Figura 1 - Mapa da região média do Rio Jequitinhonha, mostrando a ocorrência dos taxa endêmicos (adaptado de IBGE 1972). Escala 1:1.000.000. Cidades: 1. Itinga, 2. Itaobim, 3. Jequitinhonha, 4. Medina, 5. Pedra Azul. Códigos: ^ Tacinga braunii; $\boldsymbol{\Delta}$ Coleocephalocereus aureus; $\triangle$ C. purpureus; $\bigcirc P i$ losocereus cenepequei; * P. multicostatus; $\bullet P$. subsimilis. 


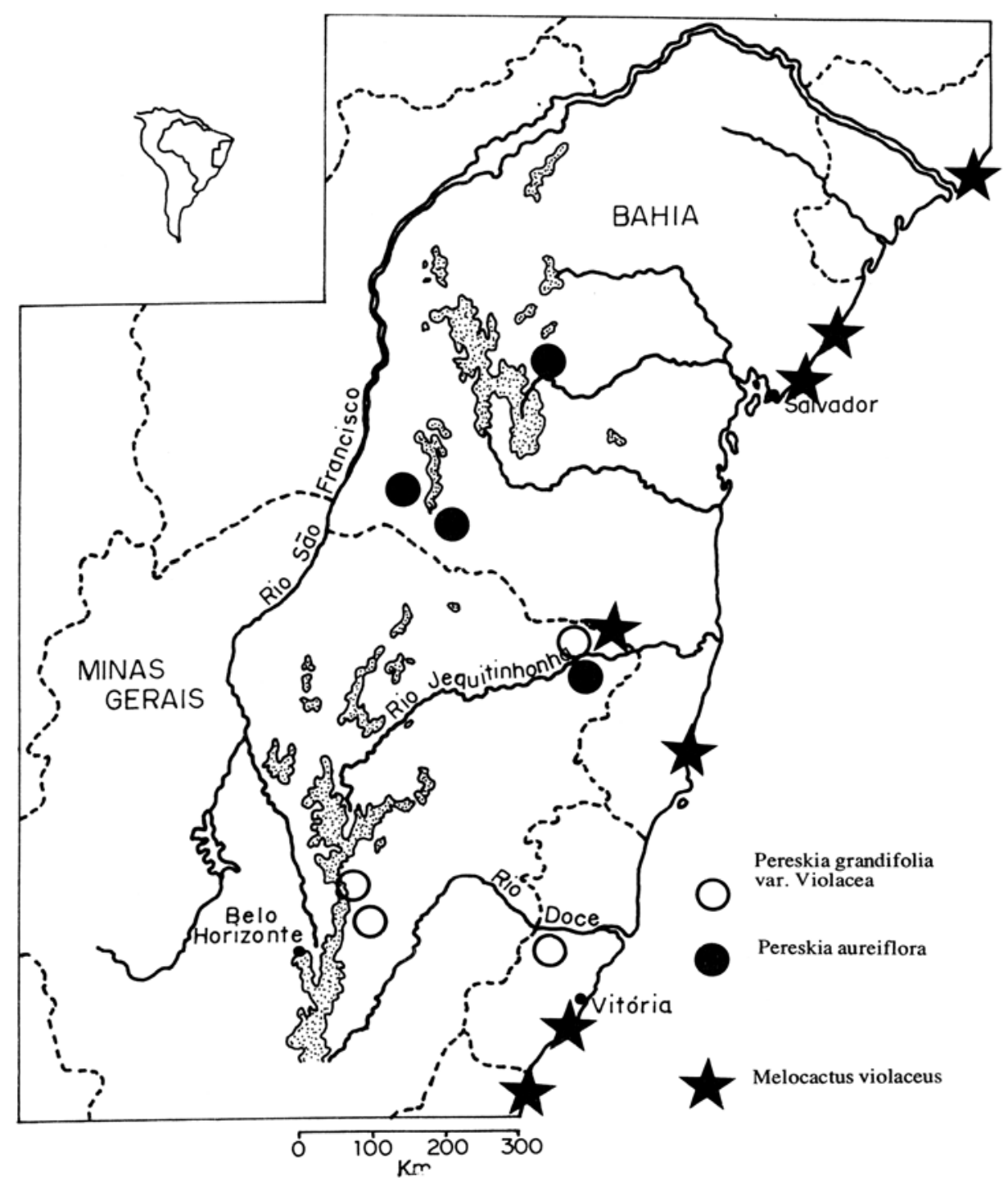

Figura 2 - Mapa da costa leste do Brasil. (adaptado de Giulietti et al., 1987), mostrando os táxons com distribuição disjunta no Brasil Oriental. 
A análise das afinidades florísticas das cactáceas ocorrentes na região possibilitou a divisão dos taxa do Vale do Rio Jequitinhonha de acordo com as seguintes categorias:

a) taxa conhecidos somente para a região do médio Jequitinhonha (Figura 1) - Dentre essas espécies, a mais notável é Tacinga braunii, muito distinta da outra espécie desse gênero, endêmico do Brasil Oriental. Pilosocereus multicostatus pode ser relacionado com espécies distanciadas geograficamente, como $P$. aurispinus, originária das margens do Rio São Francisco, na Bahia e $P$. mucosiflorus, dos afloramentos graníticos do Piauí. As demais espécies de Pilosocereus necessitam maior avaliação taxonômica para permitir suposição de correlações. Coleocephalocereus aureus e $C$. purpureus são espécies próximas, constituindo o subgênero Buiningia, exclusivo da região.

b) taxa com distribuição geográfica disjunta (Figura 2) - Com exceção de três coleções de Pereskia aureiflora feitas na Bahia, todos os outros dados relativos a esta espécie foram coletados no vale do rio Jequitinhonha, a leste e a oeste de Itaobim. a espécie mais próxima é Pereskia guamacho, originária do Norte da Venezuela (Leuenberger 1986). É interessante ressaltar que $P$. aureiflora e Pseudoacanthocereus brasiliensis ocorrem simpatricamente no Vale do Jequitinhonha, ao mesmo tempo que Pereskia guamacho e Acanthocereus (Pseudoacanthocereus) sicaraguensis Croizat \& Tamoyo, espécie próxima de Pseudoacanthocereus brasiliensis, ocorrem simpatricamente na Venezuela. Outra espécie de Pereskia, $P$. grandiflora var. violacea, típica da vegetação florestal do leste da Cadeia do Espinhaço e, notadamente do Vale do Rio Doce também foi coletada na região.

Dentre as cactáceas encontradas, talvez Melocactus violaceus, ocorrente na Serra da Areia, ao Norte da cidade de Jequitinhonha (Taylor, 1991), represente o mais extraordinário registro para a área estudada. Melocactus violaceus era, até então, tida como uma espécie de distribuição restrita às restingas litorâncas (do Rio de Janeiro até Natal), tendo sido agora encontrada no interior do continente, a cerca de $1100 \mathrm{~m}$ s.n.m., numa região de vegetação de altitude, crescendo sobre areia quartzítica.

c) taxa do Brasil Oriental com distribuição ampla, encontrando o limite Sul de ocorrência no Vale do rio Jequitinhonha - Os taxa aqui considerados são: Cereus jamacaru, Brasilicereus phaeacanthus, Malocactus ernestii, Pseudoacanthocereus brasiliensis, Arrojadoa penicillata e Opuntia sp. nov.

d) Outros taxa amplamente distribuídos:

- Brasil Oriental: Opuntia inamoena, Pilosocereus floccosus, Melocactus bahiensis ssp. amethystinus.

- Leste da América do Sul: Selenicereus setaceus, Opuntia (Brasiliopuntia) brasiliensis (= Brasiliopuntia subacarpa Rizzini \& Mattos-F.) 
Os taxa situados nas categorias (a) e (b) sugerem que a região semi-árida do médio Rio Jequitinhonha seja remanescente de uma vegetação mais extensa, e que tenha sido mantida graças a características peculiares do clima e do solo da região, podendo ser considerada como um refúgio para as cactáceas de diversos tipos de vegetação (caatinga, restinga e diversos tipos de afloramentos rochosos). Há probabilidades de que não exista outra área com semelhantes dimensões no Leste do Brasil que apresente tal concentração de taxa da família Cactaceae. Maiores investigações a esse respeito serão realizadas oportunamente.

\section{Referências Bibliográficas}

GIULIETTI, A.M.; N.L. MENEZES; J.R. PIRANI; M. MEGURO, \& M.G.L. WANDERLEY, 1987. Flora da Serra do Cipó, Minas Gerais: Caracterização e lista de espécies. Bolm. Bot, Univ. S. Paulo 9: 1-151.

HUNT, D. \& N.P. TAYLOR (eds.) 1991. Notes on Miscellaneous genera of Cactaceae. Bradleya 9:81-92.

IBGE, 1972. Carta do Brasil ao Milionésimo. P. Doc. \& Div. Geográfica e Cartográfica. SE-24, pg. 36.

KING, L.C. 1956. A geomorfologia do Brasil Oriental. Revta bras. Geogr. 18(2): 147-263.

LEUENBERGER, B.E. 1986. Pereskia (Cactaceae). Mem. New York Bot. Gard., 41 .

RITTER, F. 1979. Kakteen in Suedamerika, v.1. (Brasilien, Paraguay, Uruguay). Selbstverlag, Spangenberg.

RIZZINI, C.T. 1979. Tratado de Fitogeografia do Brasil, v. 2. Hucitec, Univ. S. Paulo, São Paulo.

TAYLOR, N.P. (1991) The genus Melocactus (Cactaceae) in Central and South America. Bradleya 9: 1-80

TAYLOR, N.P. \& D.C. ZAPPI, 1989. An alternative view of generic delimitation and relationships in tribe Cereeae (Cactaceae). Bradleya 7: $13-40$. 